\title{
Correction to: The Web Was Done by Amateurs
}

\author{
Marco Aiello
}

\section{Correction to: \\ M. Aiello, The Web Was Done by Amateurs, https://doi.org/10.1007/978-3-319-90008-7}

Some chapters in the initially published version contain a wrong affiliation of the author.

The correct affiliation of the author Marco Aiello is: University of Stuttgart, Stuttgart, Germany.

The affiliation has been corrected. 